\title{
COMMON REED BRIQUETTING MODELING WITH WORKING MODEL PROGRAMME
}

\author{
Edgars Repsa, Eriks Kronbergs \\ Latvia University of Life Sciences and Technologies, Latvia \\ edgars.repsa@1lu.lv, eriks.kronbergs@1lu.lv
}

\begin{abstract}
Nonlinear particle resistance force characteristics were experimentally obtained during compacting depending on the size of the particles. Similar nonlinear characteristics were obtained with MathCad programme using a spring incorporated in a rhomboid mechanism. The similar mechanism was used for modelling nonlinear particle resistance characteristics during the briquetting process with the Working Model programme. A hydraulic pressing mechanism on rhomboid basis with incorporated actuator was created according to the patent LV 14201 B. Working Model 2D programme lets acquire the pressing force and resistance force changing values during compacting simulation. Comparing the results of the experiments and modelling with Working Model programme the maximal difference is $5.1 \%$ for the force values. Difference between the results of modelling with MathCad and Working Model is less than $0.14 \%$ for the force values. The linear actuator force characteristic curve let easy calculate the pressing energy $1.34 \pm 0.28 \mathrm{~kJ}$ for common reed particles obtained with the hammer mill screen size $1.5 \mathrm{~mm}$. Comparing with the calculated experimentally determined pressing energy $1.46 \pm 0.31 \mathrm{~kJ}$ the difference is $0.12 \mathrm{~kJ}(8.2 \%)$.
\end{abstract}

Keywords: common reed, rhomboid mechanism, Working Model.

\section{Introduction}

Fossil energy sources are exhaustible and have substantial impacts on the natural environment and are the main reason of climate change [1;2]. Vegetation biomass likewise forestry residues can expand the existing solid biofuel resources. The use of sawdust for the production of fuel briquettes and pellets has been adequately researched [3;4] and now investigation of vegetation biofuel production processes is actual. Agricultural crop residues are mainly used for organic fertilizer production, therefore alternative biomass resources are necessary to be found.

The lake restoration process includes removal of common reed (Phragmites australis) vegetation [5;6]. This biomass is also an available resource for solid biofuel production in Latvia. The briquetting process improves the density of biofuel $\left(746-964 \mathrm{~kg} \cdot \mathrm{m}^{-3}\right)$ depending on size of particles after common reed grinding. Experimentally nonlinear particle resistance characteristics during compacting were obtained. Similar nonlinear characteristics were obtained with MathCad programme using a spring incorporated in a rhomboid mechanism. The similar mechanism was used modelling the nonlinear particle resistance characteristics during the briquetting process with the Working Model programme. A hydraulic pressing mechanism on rhomboid basis with incorporated actuator was created according to the patent LV $14201 \mathrm{~B}$. Working Model 2D programme lets acquire the pressing force and resistance force changing values during compacting simulation [7]. For the rhomboid resistance mechanism the linear characteristic of the spring resistance was used during simulation. Nonlinear characteristic of complete resistance mechanism was obtained. Comparing the results of previous compacting experiments and simulation the maximal difference $5.1 \%$ for $1.5 \mathrm{~mm}$ common reed particle compacting resistance force was obtained. The purpose of the work is the rhomboid pressing mechanism modelling with Working Model programme in order to improve the design of the press equipment. The rhomboid mechanism can be used for modelling of different size common reed particle pressing process resistance force.

\section{Materials and methods}

Laboratory compaction experiments were out in a closed die with the diameter $35 \mathrm{~mm}$ by means of laboratory hydraulic press equipment. The dosage of $35 \pm 0.1$ grams of grinded common reed was used for every briquette pressing. Maximum pressure $350 \mathrm{MPa}$ had been achieved in compacting. Average moisture content of grinded common reeds was $8.7 \%$. During the briquetting experiments the briquetting pressure was measured with a calibrated pressure sensor and pressing piston displacement with a displacement transducer. For data collection the Data Logger Pico and computer were used. Energy requirement for compacting was obtained from force - displacement curves by graphical integration. In previous studies of the compacting experiments the non-linear particle 
compression resistance characteristic with Mathcad programme was developed. The mechanical model contains a spring incorporated in the rhomboid mechanism. The Working Model 2D programme is capable of modelling the motion of rigid bodies that may be connected by a variety of joints and constraints. The rhomboid mechanism for the pressing resistance force modelling in Working Model 2D programme is shown in Fig. 1. The mechanism consists of two similar pressing links $1,1^{\prime}$ and supporting links 2, 2'. For modelling the spring 3 with linear characteristic was used. Rhomboid mechanism drive was realized with the actuator 5.

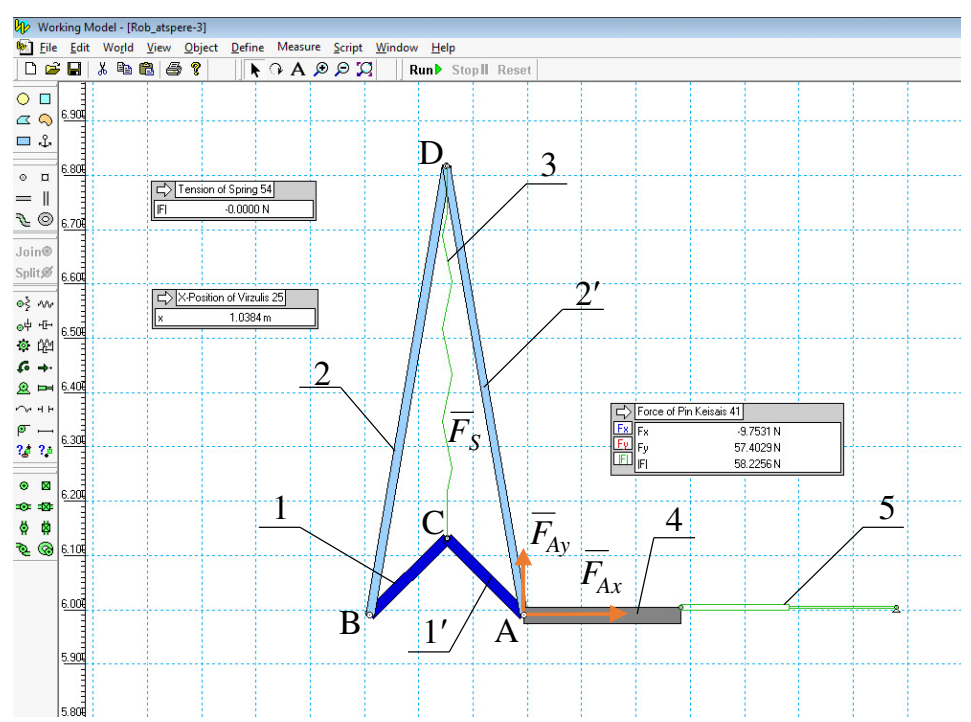

Fig. 1. Rhomboid - spring model: 1, 1' - pressing links; 2, 2' - supporting links;

3 - spring; 4 - pressing piston; 5 - actuator

During modelling the force $\bar{F}_{A x}$ in the pin A was measured. The result of the force modelling was compared with the calculation result with MathCad and experiments.

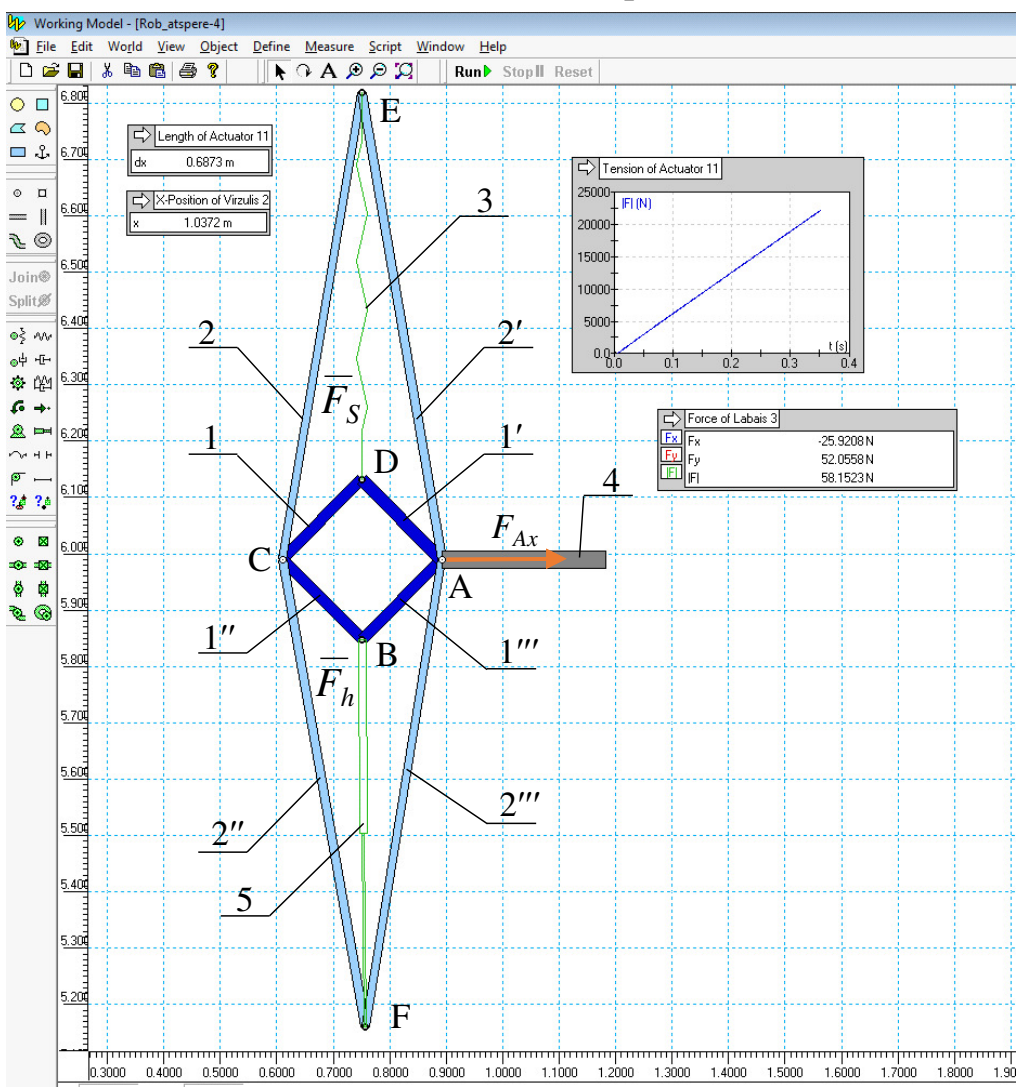

Fig. 2. Rhomboid - spring and actuator model: 1, 1', 1", 1"' - pressing links; $2,2^{\prime}, 2^{\prime \prime}, 2^{\prime \prime \prime}$ - supporting links; 3 - spring; 4 - pressing piston; 5 - actuator 
For the necessary pressing force modelling in Working Model programme a model from two rhomboid mechanisms was created (Fig. 2). The compound mechanism consists of two similar rhomboids. During modelling the pressing resistance force with rhomboid links and the linear spring 3 was simulated and the necessary pressing force from the actuator 5 was measured.

\section{Results and discussion}

During laboratory experiments the compacting force - displacement characteristics were determined. Fig. 3 shows the results of the pressing resistance modelling with Working Model programme, calculation results with MathCad programme and the results of the experiments. The experimentally obtained pressing force - displacement curve for common reed particles obtained with the hammer mill screen size $1.5 \mathrm{~mm}$ was used to evaluate the results of modelling. In the laboratory experiments obtained results show that the shapes of the force - displacement characteristics of compacting of common reed particles in the laboratory press equipment were similar nonlinear curves with two quasilinear parts. Pressing energy consumption was obtained from the force - displacement curves by graphical integration. Calculated $35 \mathrm{~mm}$ briquette pressing energy $1.46 \pm 0.31 \mathrm{~kJ}$ for common reed particles grinded with the screen size $1.5 \mathrm{~mm}$.

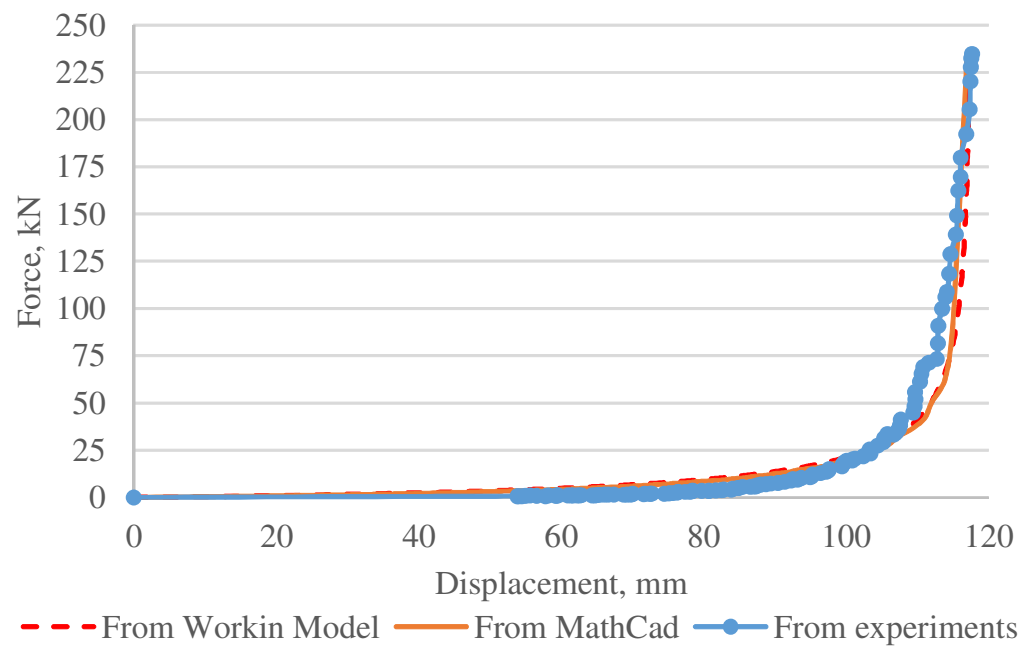

\section{Fig. 3. Force - displacement characteristic from modelling}

Comparing the results of the experiments and modelling with Working Model programme the maximal force value difference is $5.1 \%$. Comparing the results of the experiments and calculation with MathCad programme the maximal difference is $4.96 \%$. The difference between the results of both modelling modes is less than $0.14 \%$.

The modelling results for the necessary pressing force are shown in Fig. 4.

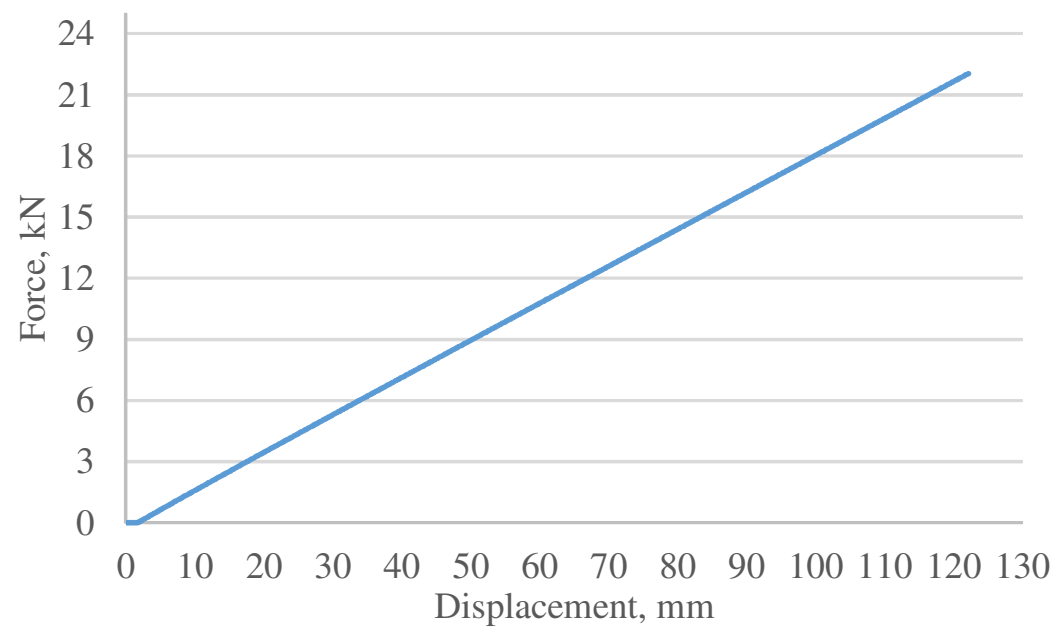

Fig. 4. Actuator pressing force from modelling 
The modelling results show that the obtained pressing force depending on the actuator displacement has a linear characteristic equal to the linear spring characteristic. The created rhomboid mechanism with incorporated spring allows modelling the nonlinear pressing resistance using the linear spring characteristic. The obtained linear relationship simplifies determination of the hydraulic drive parameters and can be used for energy calculation. The calculated actuator pressing energy was $1.34 \pm 0.28 \mathrm{~kJ}$. Comparing with the calculated experimentally determined pressing energy $1.46 \pm 0.31 \mathrm{~kJ}$, the difference is $0.12 \mathrm{~kJ}(8.2 \%)$.

\section{Conclusions}

1. The experimentally obtained $35 \mathrm{~mm}$ briquette pressing energy is $1.46 \pm 0.31 \mathrm{~kJ}$ for common reed particles grinded with the screen size $1.5 \mathrm{~mm}$.

2. Comparing the results of the experiments and modelling with Working Model programme the maximal force value difference is $5.1 \%$.

3. Comparing the results of the experiments and calculation with MathCad programme the maximal difference is $4.96 \%$.

4. The modelling results with Working Model show that the obtained pressing force depending on the actuator displacement has a linear characteristic equal to the linear spring characteristic.

5. The calculated actuator pressing energy was $1.34 \pm 0.28 \mathrm{~kJ}$. Comparing with the experimentally determined pressing energy, the difference is $0.12 \mathrm{~kJ}(8.2 \%)$.

6. The rhomboid mechanism can be used for modelling of different size common reed particle pressing process resistance force.

\section{References}

[1] Li Y., Xu H., Jing C., Jiang J., Hou X. A novel heat transfer model of biomass briquettes based on secondary development in EDEM. Renewable Energy, Volume 131, 2019, pp. 1247-1254.

[2] Muazu R.I., Stegemann J.A. Biosolids and microalgae as alternative binders for biomass fuel briquetting. Fuel, Volume 194, 2017, pp. 339-347.

[3] Denga T., Alzahranib A. M., Bradleya M. S. Influences of environmental humidity on physical properties and attrition of wood pellets. Fuel Processing Technology. Volume 185, 2019, pp. 126-138.

[4] Frodeson S., Henriksson G., Berghel J. Effects of moisture content during densification of biomass pellets, focusing on polysaccharide substances. Biomass and Bioenergy. Volume 122, 2019, pp. 322-330.

[5] Bjork S. Limnological Methods for Environmental Rehabilitation. The Fine Art of Restoring Aquatic Ecosystems. Schweizerbart Science Publishers, 2014, 381 p.

[6] Huhta A. Decorative or Outrageous - The significance of the Common Reed (Phragmitesaustralis) on water quality. Comments fromTurku University of Applied Sciences, 2009, 33 p.

[7] MSC.Software Corporation. Working Model 2D software, User's Manual (2000). 